\title{
A Hepatitis C Treatment Program Based in a Safety-Net Hospital Patient-Centered Medical Home
}

Karen E. Lasser, $M D, M P H^{1-3}$

Alexandra Heinz, LCSW, MPH

Leandra Battisti, PharmD ${ }^{3}$

Alexandria Akoumianakis, $\mathrm{CPbT}^{3}$

Ve Truong, $B S^{1}$

Judith Tsui, MD, MPH

Glorimar Ruiz, $M D^{3}$

Jeffrey H. Samet, MD, MA, MPH $H^{1-3}$

'Section of General Internal Medicine, Boston Medical Center, Boston, Massachusetts

${ }^{2}$ Boston University Schools of Medicine and Public Health, Boston, Massachusetts

${ }^{3}$ Boston Medical Center, Boston, Massachusetts

${ }^{4}$ Division of General Medicine, Harborview Medical Center, University of Washington, Seattle, Washington

Conflicts of interest: authors report none.

\section{CORRESPONDING AUTHOR}

Karen E. Lasser, MD, MPH

Section of General Internal Medicine,

Room \#2094

Boston Medical Center

Boston University Schools of Medicine and Public Health

801 Massachusetts Ave

Boston, MA 02118

Karen.lasser@bmc.org

\begin{abstract}
Hepatitis C virus (HCV) infection is a major public health problem. Urban safetynet hospitals are a prime location for HCV treatment delivery. Showing that physicians in primary care settings can deliver HCV infection care is important to expand treatment; models doing so in the era of newer oral HCV medications are needed. This article describes an innovative and successful HCV primary care treatment program in a patient-centered medical home based at an urban, safety-net hospital. The program is public health oriented in that a central team member is a public health social worker who performs population management and addresses underlying social determinants of health to facilitate engagement in HCV treatment. Other team members include general internists trained to treat HCV infections, a pharmacist, and a pharmacy technician. The program is funded with revenue generated by the $340 \mathrm{~b}$ drug discount program, which allows providers to generate revenue when patients fill prescriptions at pharmacies in safety-net settings, as insurance reimbursements for medications exceed the cost at which safety-net providers purchase medications. During the course of 1 year, the program received 302 referrals. Of these approximately 23\% have received treatment.
\end{abstract}

Ann Fam Med 2017;15:258-261. https://doi.org/10.1370/afm.2069.

\section{INTRODUCTION}

I $\mathrm{n}$ the United States, hepatitis $\mathrm{C}$ virus (HCV) infection is a major public health problem, causing 15,000 deaths annually. ${ }^{1}$ Urban safety-net hos-

pitals, serving large populations of patients with substance use disorders, are prime locations for treating $\mathrm{HCV}$-infected patients. Knowing that physicians in primary care settings can deliver care to patients infected with $\mathrm{HCV}$ is important for expanding treatment, ${ }^{2}$ and models of primary care treatment delivery in the era of newer oral HCV medications are needed.

Prior studies ${ }^{2-4}$ have demonstrated that general internists can successfully deliver HCV care for urban underserved patients in primary care settings. We are unaware, however, of primary care treatment programs in the era of newer oral medications. The simplicity of oral regimens that have few side effects and are effective across genotypes enables primary care physicians to provide treatment. Thus, these physicians may be able to prescribe newer agents with scalable treatment models. Such models would not require videoconferencing to provide specialist backup, as is the case with project $\mathrm{ECHO}$ (Extension for Community Healthcare Outcomes). ${ }^{5}$

We implemented a primary care, multidisciplinary treatment model supported by revenue from the $340 \mathrm{~b}$ drug discount program. ${ }^{6,7}$ Currently $40 \%$ of US hospitals participate in the program, which allows safety-net hospitals and health centers to generate revenue when patients fill prescriptions at their pharmacies, as insurance reimbursements for medications exceed the cost at which safety-net providers purchase medications. Such revenue can be used to support multidisciplinary staffing models to deliver treatment. Programs as the one we describe may inform policy questions regarding the future of this program and how to best target these funds. ${ }^{6,8}$ 


\section{METHODS}

The program is based in the Adult Primary Care Practice at Boston Medical Center, an accredited patientcentered medical home $(\mathrm{PCMH})$. The program includes 9 general internists who completed the American Association for the Study of Liver Diseases online training modules $^{9}$ and are thus trained to treat patients infected with HCV. Subsequent to this training, these physicians shadowed experienced specialist physicians for a single clinic session. Specialists were not co-located with the clinicians, but they were available by telephone and electronic messaging to provide clinical backup.

Primary care physicians and nurse practitioners who do not treat $\mathrm{HCV}$-infected patients refer them to the program based on laboratory notifications of positive tests, electronic health record (EHR) reports of $\mathrm{HCV}$-infected patients who are not engaged in care, and peer referrals. The multidisciplinary team includes a public health social worker (case manager), the primary care physicians trained to treat $\mathrm{HCV}$-infected patients, a pharmacy technician, and a pharmacist.

\section{Previsit Activities}

The case manager reviews charts and calls patients if the chart shows viremia, no engagement in care of HCV infection, and no comorbidities requiring specialty treatment. Infectious diseases physicians treat patients co-infected with human immunodeficiency virus, and gastroenterologists treat patients co-infected with hepatitis B virus, those with decompensated cirrhosis, and those with major comorbidities (eg, advanced renal insufficiency). The case manager explains the purpose of the referral and schedules appointments with treating physicians. The case manager also provides appointment reminder calls, troubleshoots insurance and transportation issues, and maintains a log to manage the patients from referral to discharge.

\section{Physician Visit 1}

The physician ascertains infection and treatment history and obtains a substance use history to assess risks of nonadherence and reinfection. The physician then counsels patients on avoiding alcohol and reducing transmission risk.

\section{Case Manager Follow-up}

When possible, the physician pages the case manager to meet the patient. The case manager assesses income, housing, legal issues, substance use, mental health, and transmission risk factors. He or she also provides education about HCV and the treatment program. The case manager then schedules follow-up to address psychosocial needs and refers, as needed, for psychiatry and counseling services, substance use treatment, tran- sitional assistance, housing resources, transportation, social security, and job training.

\section{Physician Visit 2}

The physician reviews test results and discusses treatment. He or she assesses motivation for treatment and substance use. If the patient is motivated for treatment, and substance use does not appear to be a barrier to adherence, the physician prescribes the indicated agent. Patients receive hepatitis A and B vaccines as needed.

\section{Pharmacy Technician}

For patients who are treatment candidates, the technician prepares prior authorization paperwork. If prior authorization is denied, the technician appeals. If the appeal is denied, the case manager advises the patient to request a reevaluation in 1 year.

\section{Pharmacist}

Patients who have had treatment approved meet with the pharmacist for a teaching visit. The pharmacist provides education about medication administration and the importance of adherence, adverse effects, and monitoring. The pharmacist contacts the patient 2 weeks later by telephone to assess for adverse reactions and to remind the patient about the next medication refill. The patient meets with the pharmacist at 4 weeks and at the end of treatment to discuss adherence and adverse effects, as well as for laboratory testing.

\section{Physician Visit 3}

The physician sees the patient 3 months after treatment completion and checks HCV RNA to ascertain whether a sustained virologic response has been achieved, a proxy for cure. ${ }^{10}$

We reviewed the case manager's tracking logs and the EHRs for patients from March 2015 to April 2016. We analyzed demographic, laboratory, and radiologic data. The Boston Medical Center/Boston University School of Medicine institutional review board approved the study.

\section{RESULTS}

Among 302 patients referred for treatment, threefourths were male; more than one-half were white. The mean age was 45 years; most had Medicaid. Among patients who received the respective testing, most (163 [71\%]) had HCV genotype 1, and 51 (49\%) had earlystage fibrosis (F0-F1).

Figure 1 displays the cascade of patient care. Approximately one-half of patients (157 of 302) referred for treatment in primary care attended an initial physician visit. The case manager could not contact 
Figure 1. Flowchart showing care of patients with hepatitis C virus infection in primary care.

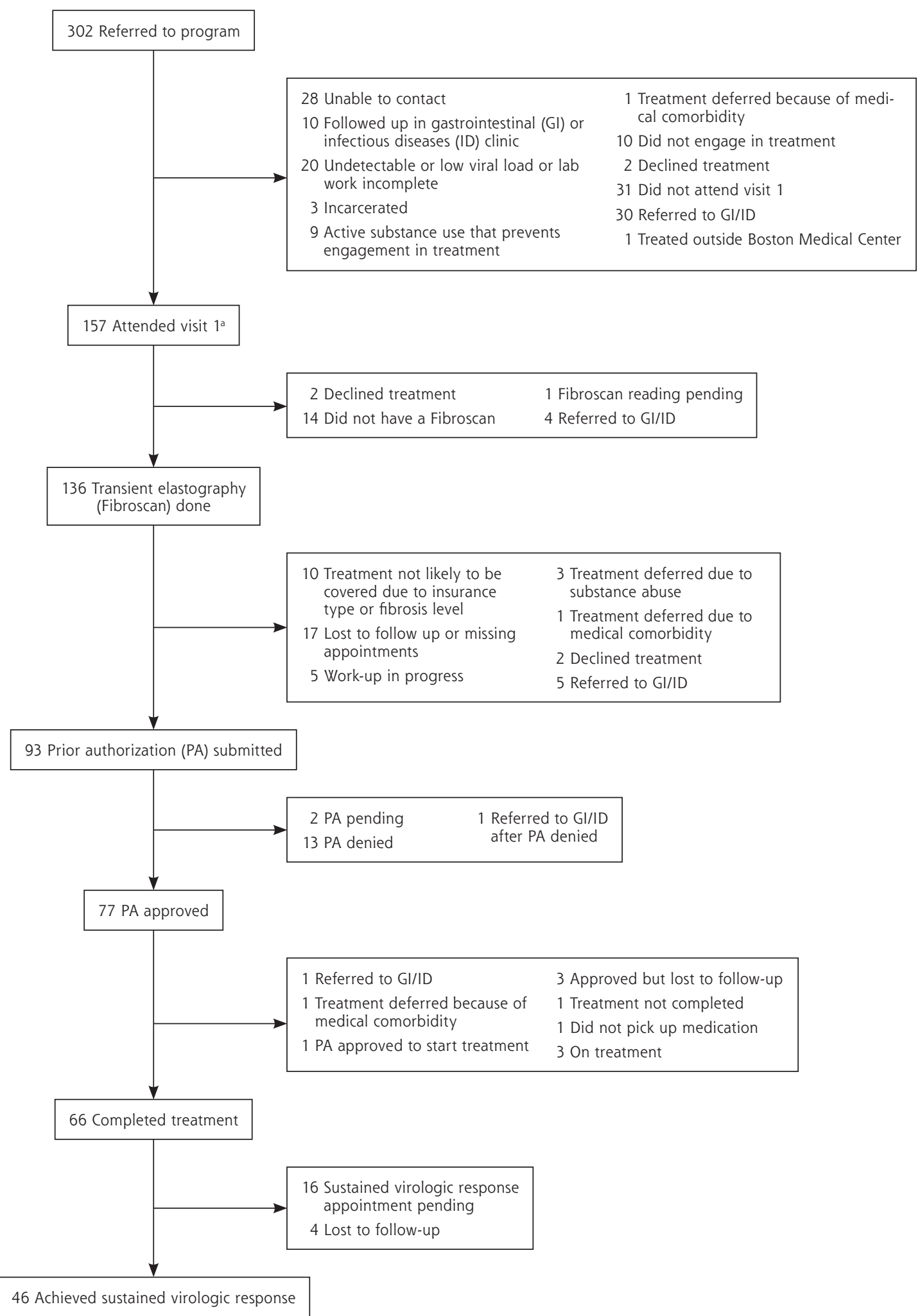

Visit 2 with physician not included in figure because visit 2 was added midway through the program. 
$9 \%$ of patients (28 of 302). Sixteen percent of patients (31 of 188) with scheduled appointments missed their first appointment. Thirteen percent of patients (40 of 302) were immediately referred to a specialist or were already engaged in treatment. Among 136 patients who completed a transient elastography to estimate fibrosis, the technician submitted a prior authorization for 93 (68\%). Of those 93 patients, 69 (74\%) continued or completed treatment. All 46 patients who attended a visit 3 months after completing treatment achieved a sustained virologic response.

\section{DISCUSSION}

This primary care treatment program for patients infected with HCV shows promise for curing selected patients. This program is unique in 2 ways; it is situated in a PCMH based in a safety-net hospital and uses a multidisciplinary approach with primary care staff working at the top of their license (that is, each team member practices to the full extent of their education and training instead of performing tasks that could be completed by another team member). Although our model employed general internists, we believe family physicians could play a similar role. Additionally, physicians' assistants and nurse practitioners could evaluate patients for HCV infection under physician supervision. Finally, we believe this model has transferability beyond large urban academic medical centers. Community health centers, for example, may have $340 \mathrm{~b}$ pharmacies and are accustomed to delivering multidisciplinary care.

Key to the program's success is the central role of a public health social worker, who performs many patient navigation functions (eg, addressing insurance barriers and transportation, and linking patients to services). Despite receiving enhanced social work services, many patients who were referred failed to engage in treatment. We suspect that substance use may have prevented patients from engaging in care, even though our program is co-located with a model, office-based, addiction treatment program. ${ }^{11}$ Those patients who miss appointments and do not engage in treatment may need additional support.

Our program may have limited generalizability. Massachusetts has the broadest Medicaid coverage of HCV treatment among the states, and it does not restrict treatment to patients with advanced fibrosis or in specialty settings. Our staffing model was funded by $340 \mathrm{~b}$ program revenue. As of August 2016, the 340b benefit no longer covers several of the most commonly used oral agents supported by Massachusetts Medicaid plans, because such plans now have contracts with drug manufacturers. Nevertheless, the program has continued since August 2016. As our institution transitions to become a Medicaid Accountable Care Organization, it is possible that the most vulnerable patients will continue to receive the support of the multidisciplinary team, as it has shown clinical benefit. Given the opportunity to design an HCV treatment program within primary care, this model, embedded in an urban safety-net hospital $\mathrm{PCMH}$, achieved impressive outcomes and has the potential for dissemination.

To read or post commentaries in response to this article, see it online at http://www.AnnFamMed.org/content/15/3/258.

Key words: hepatitis C, chronic; primary health care

Submitted September 19, 2016; submitted, revised December 12, 2016; accepted December 30, 2016.

Funding support: NIDA P30 DA040500-01-pilot grant, "Center for Health Economics of Treatment Interventions for Substance use Disorder, HCV, and HIV."

\section{References}

1. Ly KN, Hughes EM, Jiles RB, Holmberg SD. Rising mortality associated with hepatitis C virus in the United States, 2003-2013. Clin Infect Dis. 2016;62(10):1287-1288.

2. Miller L, Fluker SA, Osborn M, Liu X, Strawder A. Improving access to hepatitis $C$ care for urban, underserved patients using a primary care-based hepatitis C clinic. J Natl Med Assoc. 2012;104(5-6):244-250.

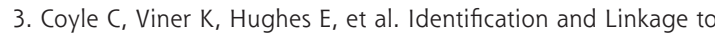
Care of HCV-Infected Persons in Five Health Centers - Philadelphia, Pennsylvania, 2012-2014. Morbidity and Mortality Weekly Report (MMWR) [CDC]. Centers for Disease Control and Prevention. 2015;64(17):459-463.

4. McGinn TG, Gardenier D, McGinn LK, et al. Treating chronic hepatitis $C$ in the primary care setting. Semin Liver Dis. 2005;25(1):65-71.

5. Arora S, Thornton K, Murata G, et al. Outcomes of treatment for hepatitis C virus infection by primary care providers. N Engl J Med. 2011;364(23):2199-2207.

6. Young K. $340 B$ discount drug program guidance is unveiled. The Commonwealth Fund. http://www.commonwealthfund.org/publications/newsletters/washington-health-policy-in-review/2015/aug/ aug-31-2015/340b-discount-drug-program-guidance-is-unveiled. Published Aug 28, 2015. Accessed Sep 25, 2015.

7. Health Resources and Services Administration. 340B Drug Pricing Program. http://www.hrsa.gov/opa/. Accessed Nov 27, 2016.

8. Wynne B. The coming storm over the $340 \mathrm{~B}$ Rx drug discount program. Health Affairs. http://healthaffairs.org/blog/2014/05/06/thecoming-storm-over-the-340b-rx-drug-discount-program/. Published May 6, 2014. Accessed Sep 25, 2015.

9. American Assocation for the Study of Liver Diseases. ACT-First: Free, online CME course for primary care providers. HVCAdvocate. http://hepatitisc.hcvadvocate.org/2014/05/act-first-free-online-cmecourse-for.html. Published May 2, 2014. Accessed Apr 29, 2016.

10. American Assocation for the Study of Liver Diseases. HCV guidance: Recommendations for testing, managing and treating hepatitis $C$. AASLD. http://www.hcvguidelines.org/full-report-view. Accessed May 17, 2016.

11. Alford DP, LaBelle CT, Kretsch N, et al. Collaborative care of opioidaddicted patients in primary care using buprenorphine: five-year experience. Arch Intern Med. 2011;171(5):425-431. 